\title{
Comunicación

\section{El relativismo teórico en comunicación. Entre la comunicación como principio explicativo y la comunicación como disciplina práctica}

Theoretical relativism in communication. From communication as a principle for explanation to communication as a practical discipline

CARLOS VIDALES GONZÁLES ${ }^{1}$

El artículo propone al relativismo teórico como una problemática actual de los procesos de producción teórica y de formación académica en los estudios de la comunicación. Se plantea un segundo orden de observación que supone mirar a la comunicación desde la propia disciplina a través de una matriz teórica general que sintetice la discusión en dos rutas posibles: la consideración de la comunicación como principio explicativo y la consideración de la comunicación como disciplina práctica.

PALABRAS ClAVE: Teoría de la comunicación, epistemología, campo de la comunicación, historia de la comunicación, cibersemiótica.
This article argues that theoretical relativism is an obstacle in current processes of theory development within communication studies. It is suggested that as a scholarly field, communication should itself be understood from a communicational perspective. A theoretical matrix is put forward indicating that communication should be considered as an explicative principle and as a practical discipline.

KEY WORDS: Communication theory, epistemology, communication field, history of communication, cybersemiotics.

1 Universidad de Guadalajara, México.

Correo electrónico: morocoi@yahoo.com

DECS, Paseo Poniente 2093, Jardines del Country, C.P. 44210, Guadalajara Jalisco, México. 


\section{INTRODUCCIÓN}

A cinco décadas de la famosa discusión entre Bernard Berelson (1959), para quien el campo de la comunicación moría, y Wilbur Schramm, Riesman y Baver (1959), para quienes dicho campo recién estaba naciendo, han aparecido una gran cantidad de trabajos que permiten avanzar en la discusión y elegir el camino hacia uno u otro lado. Sin embargo, aún no es posible rechazar la hipótesis con la que John Durham Peters (1986) resumía la discusión a finales de los años ochenta, es decir, el hecho de que la comunicación ha sido definida administrativamente pero no conceptualmente, por lo que la teoría fracasa como principio de definición, como fracasa el intento por determinar a la comunicación como un objeto claramente diferenciable de otros objetos de estudio. Por lo tanto, no se trata únicamente de recuperar las viejas discusiones, sino de explorar las consecuencias que tiene para los estudios de la comunicación en la actualidad el haberlas ignorado en los propios procesos de construcción teórica y en la práctica de investigación.

Si bien los estudios de la comunicación han centrado su atención en varios objetos de estudio, la reflexión sobre los marcos teóricos, metodológicos y epistemológicos sobre los que se basa su práctica de investigación, no han sido objeto de reflexión suficiente. El resultado del gran desequilibrio que existe entre la investigación teórica y la que puede ser llamada genéricamente como "investigación aplicada", es una suerte de relativismo conceptual, el cual acepta que es posible tener un número infinitamente grande de distintos sistemas de representaciones para presentar y representar los hechos en la realidad social (Searle, 1997), pero niega la libertad conceptual, dado que toda teoría supone de entrada un sistema conceptual y un principio constructivo dentro del cual opera su propia lógica explicativa y de formalización del mundo fenoménico que pretende explicar. En consecuencia, el relativismo conceptual implica la ruptura del sistema conceptual o de los principios constructivos dentro de los cuales opera toda formulación explicativa, dado que todo concepto cobra sentido dentro de un marco teórico concreto, por lo que su separación de dicho marco tiene como efecto inmediato la pérdida de su carga significativa (Vidales, 2009b, 2010). 
El problema del relativismo conceptual conduce entonces, de manera más general, a un relativismo teórico. De este segundo concepto los trabajos de James Anderson (1996), Jennings Bryant y Dorina Miron (2004) son un gran ejemplo, dado que, al analizar la constitución teórica del campo de estudio de la comunicación a través de los productos académicos (artículos y libros), documentan varios cientos de nombres de teorías de la comunicación, muchas de las cuales no son propiamente teorías sino propuestas axiomáticas o esquematizaciones de sistemas conceptuales. ${ }^{2}$ En este punto es importante reconocer, como lo hace el constructivismo de Humberto Maturana y Francisco Varela (2006), que "no vemos que no vemos". Los trabajos de la comunicación voltean constantemente a ver la realidad social en busca de objetos de estudio, pero rara vez voltean a ver sus propios procesos de producción de conocimientos, sus propios marcos epistemológicos, los supuestos ontológicos sobre la comunicación que se encuentran en la base de su práctica de investigación, los efectos que la elección de sus métodos de recolección de datos tienen en sus propios objetos de estudio, las repercusiones que los propios investigadores, como observadores, tienen sobre la realidad social que estudian. Es decir, pocas veces se detienen a pensar en cómo es que observan y a través de qué lo hacen, en la validez de sus principios teóricos o en la inexistencia de ellos.

Este primer escenario muestra un problema en el que deben ser distinguidos por lo menos dos niveles. En el primero se encuentra la crítica de Bernard Berelson (1959) a la fundamentación conceptual de una empresa científica, es decir, el tema es la construcción teórica en los estudios de la comunicación. Un segundo nivel tiene que ver con

2 Dentro de esta problemática, un dato importante que muestra el trabajo de Bryant y Miron (2004) es la naturaleza del uso de las teorías en el campo de estudio de la comunicación, el cual se restringe a una mera referencia, dado que en 1,806 artículos (576 relacionados con la comunicación de masas) analizados en tres revistas importantes (Journalism \& Mass Communication Quarterly, Journal of Communication y Journal of Broadcasting \& Electronic Media) encontraron 1,393 referencias hechas a 604 teorías diferentes. De las teorías encontradas, los autores reconocen que $48 \%$ de ellas fueron usadas como mera referencia. 
los efectos de la crítica de Berelson en lo que a la cientificidad de la comunicación se refiere, es decir, se trata del estatuto disciplinar de la comunicación más que del proceso de construcción teórica. Estamos entonces frente a una discusión que emerge con el nacimiento mismo de los estudios de la comunicación que integra dentro de sí la carencia de fundamentación conceptual y la cual influye directamente en la imposibilidad de establecer a la mirada comunicativa como una mirada científica y una disciplina particular.

En este sentido, si bien la historia y el debate sobre la producción teórica en los estudios de la comunicación no han sido objeto de reflexión sistemática, sí lo ha sido la reflexión sobre la comunicación como disciplina, una historia recuperada recientemente por Robert $\mathrm{T}$. Craig (2008b) en el marco de la publicación de la International Encyclopedia of Communication, un último eslabón de una larga historia. En su propuesta, Craig argumenta que el estatus de la comunicación como una disciplina o como campo interdisciplinar ha sido debatido desde los años ochenta. El Ferment in the Field que fue un número especial del Journal of Communication de 1983, estuvo principalmente centrado en la emergencia de los estudios culturales críticos y la economía política como dos frentes que se contraponían a la tradición establecida del funcionalismo de la comunicación de masas (Gerbner, 1983). El tema primario implicado por el título y los contenidos del número especial, aunque no era aceptado con el mismo entusiasmo por todos los participantes, tenía unidad en la diversidad. "Los disidentes estaban ahora 'en' el campo. El campo sería redefinido para incluirlos" (Craig, 2008b, p. 684). Por otro lado, el mismo Craig argumenta que el espíritu de inclusión estaba de alguna manera en tensión con un segundo tema también presente en el Ferment in the Field, el cual implicaba que la búsqueda de la unidad en la diversidad de los estudios de la comunicación era en realidad la evidencia de que estábamos frente a una disciplina académica distinta más que frente a un área interdisciplinar. Estas discusiones ya anunciaban los elementos de un modelo científico de la comunicación que comenzaba por posicionar a la comunicación como una disciplina de las ciencias sociales. Sin embargo, si bien el modelo se extendía a través de diversas áreas académicas y acercamientos teóricometodológicos, afirmaba la existencia de, o al menos el potencial para, 
una disciplina de la comunicación marcada por métodos característicos, líneas de investigación particulares y teorías científicas específicas. Según Craig (2008b), el modelo científico de la comunicación describía la disciplina de acuerdo a cinco criterios particulares:

(1) su origen histórico en el movimiento de la investigación interdisciplinar de la comunicación en la mitad del siglo veinte, (2) su rápido crecimiento y consolidación institucional en la última década del mismo siglo, (3) su identidad central como una ciencia social empírica, (4), su lugar apropiado como una disciplina "variable" abarcando diferentes "niveles de análisis" en el esquema de las disciplinas académicas y, (5) su urgente necesidad de re-unir la "separación" entre la comunicación interpersonal y la masiva, la cual constituye la barrera más seria para el desarrollo de un centro teórico de niveles cruzados [cross-level] en la disciplina (p. 685).

Según Craig (2008b), si bien la ciencia de la comunicación aún no tiene un centro teórico completamente desarrollado, los distintos acercamientos que hace a los mensajes fue considerado como un elemento capaz de proveer un marco general para la construcción de nuevas teorías para explicar cómo los mensajes cumplen funciones específicas a través de rangos micro y macro en los distintos niveles de análisis de la comunicación. Así, el modelo científico de la comunicación anunciaba las bases para la inclusión y la integración de la comunicación interpersonal y la masiva; sin embargo, su identidad central como una ciencia social empírica tendía a marginalizar los estudios críticos y humanistas cuya entrada masiva a los estudios de la comunicación había producido precisamente ese "fermento" en el campo. El problema radicaba en que no todos los académicos estaban de acuerdo en que la comunicación podía o debería convertirse en una disciplina independiente.

Una década después, en 1993, el Journal of Communication revisó nuevamente el problema del estatus disciplinario de la comunicación en dos números especiales titulados The Future of the Field; sin embargo, los 48 artículos presentados no revelaron ningún consenso. Según Craig, muchos escritores se referían casualmente a "la disciplina" como si no hubiera ningún cuestionamiento sobre la identidad o el estatus disciplinar, mientras algunos otros argumentaron, en ocasiones enfática- 
mente, que el campo de la comunicación no era una disciplina, aunque su actitud variaba enormemente sobre éste hecho y sobre qué hacer al respecto. En palabras de Craig (2008b)

Algunos otros fueron optimistas sobre el hecho de que el campo estaba emergiendo a través del estatus disciplinar; otros estaban seguros que algo así no estaba sucediendo. Algunos vieron la fragmentación continua del campo como un problema; otros celebraron la fragmentación como una fuente invaluable de fuerza adaptativa. Algunos llamaron urgentemente por esfuerzos para definir intelectualmente el centro de la disciplina; otros insistieron igualmente de forma urgente que cualquier esfuerzo por definir un núcleo teórico no sólo sería inútil, sino contraproducente. Además, otros fueron poco claros sobre la posibilidad o el deseo de convertirse en una disciplina, sin embargo, propusieron varias definiciones conceptuales del campo de la comunicación (p. 686).

Actualmente, como el mismo Craig argumenta, ninguna de estas visiones domina hoy en día el campo de la comunicación a comienzos del siglo XXI, dado que la desconexión entre la investigación de la comunicación interpersonal y masiva sigue siendo considerada un problema, lo mismo que el continuo crecimiento institucional del campo sin ningún consenso sobre un núcleo teórico y una epistemología científica rigurosa. En síntesis para Craig (2008b), "La visión pluralista del 'diálogo de paradigmas' también continua siendo problema así como los intentos por definir un núcleo teórico disciplinar que todavía se pueda acomodar al pluralismo del campo" (p. 686). Como es posible observar, la importancia de recuperar la discusión sobre la naturaleza epistemológica de la comunicación y de convertir su proceso de construcción teórica en un objeto de estudio, radica no sólo en la comprensión de cómo es que opera y funciona en un momento sociohistórico particular, sino en la propuesta de cómo podría funcionar, en la formulación de escenarios de lo posible, escenarios en ningún momento normativos.

$\mathrm{Y}$ en eso precisamente consiste el presente artículo, en un intento por mostrar los problemas actuales que enfrenta el campo de estudio de la comunicación cuyas raíces se encuentran en el comienzo mismo de su propia historia, es decir, se trata de recuperar los viejos debates y 
de hacer visibles algunas rutas que ya se han propuesto como vías para construir, desde ahí, nuevas preguntas y bosquejar también algunas respuestas provisionales. Por lo tanto, el artículo que aquí se presenta muestra las problemáticas actuales que han emergido en los procesos de producción teórica en los estudios de la comunicación y en los procesos de formación académica a través de la necesidad de plantear un segundo orden de observación que supone mirar a la comunicación desde la propia comunicación; un proceso desde donde se puede reconocer dos rutas posibles: su consideración como principio explicativo y su valoración como disciplina práctica.

\section{LAS IMPLICACIONES PRÁCTICAS DE (RE)PRODUCIR LOS PROBLEMAS TEÓRICOS EN LOS ESTUDIOS DE LA COMUNICACIÓN}

En el discurso de inauguración del encuentro anual de la International Communication Association en 2005, su presidente en turno, Wolfgang Donsbach, argumentaba que todo intento por dar cuenta del estado de una disciplina es necesariamente limitado debido a la velocidad con que crece su producción académica como es el caso de los estudios de la comunicación. En este sentido, apuntaba Donsbach (2006), pese a que la comunicación como campo de investigación ha visto el mayor crecimiento de probablemente todas las áreas durante los últimos 30 años, éste aún carece y pierde identidad con el paso del tiempo, inclusive a pesar de los debates que ponían al centro la pregunta por saber si la comunicación era o no una disciplina. Para Donsbach, algunos sostienen que la comunicación más que una disciplina es un campo con un objeto compartido, debate que surgió con el nacimiento mismo de los estudios de la comunicación, desde donde también se han propuesto como contra-argumentos que la comunicación es en realidad una "ciencia integrativa", una "ciencia sinóptica" o bien, una "interdisciplina", aunque en realidad ninguno de ellos resuelve el problema de la identidad. 3

3 Esta misma problemática ha sido reconocida por Susan Herbst (2008), quien argumenta que pese a que se han fundado revistas especializadas y asociaciones sobre comunicación, en realidad seguimos importando marcos 
Por otro lado, pese a que Donsbach reconoce que los estudios de la comunicación han acumulado mucha y muy buena evidencia empírica de los procesos de comunicación, también asume que sufren crecientemente de "erosión epistemológica" promovida por los desarrollos dentro y fuera del campo de estudio. Desde dentro es un renacimiento de acercamientos que se abstienen de poner sus hipótesis a prueba y desde fuera son un incremento en la competencia de todos los académicos del campo con gente no académica de todo tipo. Sin embargo, más importante aún es su reconocimiento de que los estudios de la comunicación tienen un conocimiento lógico y preciso en muchas áreas pero se tiende a perder orientación normativa en la investigación empírica. Desde su punto de vista, hacer investigación empírica sin metas normativas se puede convertir fácilmente en una actividad arbitraria, irrelevante y aleatoria (Donsbach, 2006).

Por lo tanto, no se trata únicamente de intentar bosquejar nuevas respuestas a interrogantes no resueltas, sino de comprender las consecuencias que ha tenido para los estudios de la comunicación caminar hacia adelante en su práctica de investigación sin haber resuelto las preguntas con las que nace, de seguir construyendo conocimiento sobre una base poco clara. El resultado es lo que Robert T. Craig (1999) ha llamado las "rutas de la incoherencia", lo que Wolfgang Donsbach (2006) llama la "erosión epistemológica", lo que Jeffrey St. John, Ted Striphas y Gregory Shepherd (2006) han llamado el "pluralismo teórico indiferenciado" y lo que aquí se ha reconocido como el "relativismo teórico", todos términos que describen una misma problemática y que llevaron a teóricos como Klaus Krippendorff (1994) a plantear una crítica hacia la construcción teórica en el campo de la comunicación contrastada con lo que él mismo denomina la explicación reflexiva, la cual, más allá de acortar las fronteras de la investigación, invita a una tarea de reconstrucción del propio campo.

De igual forma, St. John et al. (2006) se posicionan en contra de la idea del pluralismo teórico y de la creencia de que las teorías tienen el

teóricos de otras disciplinas para nuestros propios estudios, lo cual se hace porque si uno quiere demostrar que es un campo importante, entonces tiene que hablar el lenguaje de las otras ciencias o disciplinas para probar la propia legitimidad. 
mismo mérito si las entendemos y apreciamos en sus propios términos, por lo tanto, anuncian la necesidad de jerarquizarlas, dado que hay una diferencia entre la construcción de teorías y la aplicación de las mismas, es decir, los autores manifiestan un rechazo explícito de las afirmaciones contemporáneas sobre el estudio de la comunicación en el sentido de que un pluralismo teórico indiferenciado es algo bueno. De lo anterior deviene el reconocimiento de dos puntos de coincidencia que aquí se tiene con los autores. El primero tiene que ver no sólo con el pluralismo teórico indiferenciado, sino con la cantidad de productos que se presentan como teóricos. La vasta producción pareciera confirmar la idea de que lo producido en verdad está influenciando de alguna manera y "en algún lugar" al mundo en el que vivimos, al tiempo que fortalece un espacio académico, pero quizá lo que suceda sea un proceso inverso. El segundo punto tiene que ver con el legado del empirismo y las formas de normalizar la producción teórica. En este punto se podría argumentar que la reproducción de un tipo específico de hacer teoría no sólo reproduce un esquema de escritura sino que propone uno similar para la producción/construcción teórica.

Por otro lado, una segunda problemática relacionada con el pluralismo teórico es la que ya había hecho explícita James Anderson (1996) en su trabajo evaluativo del nivel ontológico, epistemológico, praxeológico y axiológico de las teorías de la comunicación; trabajo que partía del reconocimiento de dos problemas específicos. El primero es producido por la virtual destrucción de la epistemología tradicional en las últimas décadas (Wallerstein, 2005), lo que ha tenido como principales consecuencias que las observaciones sean consideradas argumentos, las teorías prácticas sociopolíticas y que la verdad sea plural. El otro problema es el carácter politizado de cualquier posición epistemológica junto con los métodos de enseñanza en la educación (principalmente de posgrado), es decir, en el fondo hay un interés por mostrar algunas de las prácticas por las que los sujetos se convierten en ciudadanos naturales de alguna comunidad intelectual. La relación entre ambos problemas genera un escenario con características peculiares. Se producen textos académicos que suponen una formación académica compartida y un acuerdo en la construcción de los sistemas conceptuales, aparecen así los mismos conceptos en contextos teóricos diferenciados que los 
vuelven incoherentes o carentes de sentido. El móvil es la construcción argumentativa que se mueve en dos sentidos, uno que tiene que ver con la comprobación de algo que ya se sabe que sucede a través de algún principio teórico y otro que tiene que ver con la producción de constructos teóricos que son formulados de acuerdo a un problema específico y bajo la consigna de que deben ser verdaderos.

Ambos procesos tienen como objetivo principal no la producción de conocimiento o de un principio teórico, sino la normalización ${ }^{4}$ de la argumentación que se presenta sobre un problema dado. La normalización complejiza enormemente el estudio de la teoría, puesto que todo análisis tiene que reconocer que la teoría no es sólo un conjunto de proposiciones textuales así denominadas, sino prácticas continuas de comunidades intelectuales. Al exponerse por sí mismos a este cúmulo de actividades prácticas, los estudiantes están también inmersos, generalmente sin su consentimiento razonado o informado, en modos particulares de pensamiento, los cuales devienen de los "métodos de adoctrinamiento" (indoctrinating methods) identificables en las prácticas docentes. De esta forma, el estudio de los métodos no sólo induce al estudiante dentro de una ideología académica, sino también dentro de

4 Anderson (1996) utiliza el concepto de normalización en el sentido que Thomas Kuhn le de al concepto de "ciencia normal". En palabras de Khun (2006), "el éxito de un paradigma en sus momentos iniciales consiste en gran medida en una promesa de éxitos detectable con ejemplos seleccionados y aún incompletos ... La ciencia normal consiste en la actualización de dicha promesa, actualización que se logra extendiendo el conocimiento de aquellos hechos que el paradigma exhibe como especialmente reveladores, aumentando la medida en que esos hechos encajan con las predicciones del paradigma, así como articulando más aún el paradigma mismo" (p. 89). En este sentido, "la investigación en la ciencia normal se orienta a la articulación de los fenómenos y teorías ya suministrados por el paradigma" (p. 90). En este caso en particular, la normalización hace referencia al proceso en el que una determinada argumentación busca identificarse con algún principio teórico precedente con la finalidad de validarse. Como puede observarse, el fin no es la producción de conocimiento sino la reproducción del conocimiento ya existente. 
dominios teóricos específicos y, sobre todo, dentro de una comunidad científica particular (Anderson, 1996).

Lo anterior implica que la construcción de conocimiento se encuentra determinada en primera instancia por mecanismos que han regulado y normalizado la producción académica dentro de una fórmula que permite la destrucción de los sistemas conceptuales o inhibe su construcción en beneficio de la (re)producción de un quehacer científico, lo cual ha generado no sólo que los problemas de la fundamentación epistemológica no sean recuperados, sino que su recuperación sea irrelevante. Por lo tanto, quizá la idea no sea la elaboración de una respuesta definitiva, sino la contrastación de la evidencia ya recuperada. Por ejemplo, en su presentación a la segunda edición del Handbook of Communication Sciences, Charles R. Berger, Michael E. Roloff y David R. Roskos-Ewolden (2010) reconocen la necesidad, no de recuperar los debates anteriores, sino de confrontar las propuestas existentes, dado que la confrontación empírica (de teorías) es precisamente un signo de la vitalidad y la dinámica de una empresa científica, por lo que los debates que las propias confrontaciones generan son de suma importancia para el avance de toda disciplina. Pero también reconocen que incluso en una etapa temprana, los investigadores dentro de los estudios de la comunicación han mostrado muy poca evidencia de estas controversias teóricas y académicas.

Por lo tanto, si el campo de la comunicación es altamente diverso en métodos, teorías y objetos de estudio "¿Qué es, si es que lo hay, lo que une al campo como una entidad coherente?" (Craig, 2008b, p. 675). Este es un punto clave sobre el que Robert T. Craig llama la atención al suponer que si la comunicación es el foco que une todas esas teorías, objetos o métodos, entonces cabe preguntarse,

¿Pero cuál es la naturaleza de ese foco común? ¿Es la comunicación sólo un tema nominal que débilmente une una serie de profesiones y disciplinas que de otra forma no estarían relacionadas? ¿Es la comunicación verdaderamente un campo interdisciplinar en el que el progreso del conocimiento es sólo posible a través de la cooperación cercana y sinergia entre las varias y distintas disciplinas que componen el campo? ¿Es la comunicación de hecho (además de su aparente fragmentación), o por lo menos potencialmente, el objeto de 
una disciplina intelectual distinta en su propio derecho? ¿Puede ser en algún respecto verdadera alguna de estas interpretaciones sobre el campo? (p. 675).

Para Craig (2008b) los estudios de los medios y la comunicación emergieron más o menos de forma independiente de muchas otras fuentes, por lo que la formación del campo de la comunicación ha resultado de la convergencia parcial de varias disciplinas y líneas de investigación que se intersecan de forma compleja, todas relacionadas de alguna manera con el fenómeno de la comunicación, pero nunca han sido estrechamente integradas como un cuerpo coherente de conocimiento. Por lo tanto, la manifiesta diversidad de la investigación de la comunicación no es un desarrollo reciente, sino algo que ha caracterizado al campo a través de toda su historia. Las tradiciones intelectuales que hoy conforman al campo provienen de las humanidades y las ciencias sociales, donde la comunicación fue un tema compartido hasta su institucionalización, para la cual importó principios explicativos de las ciencias sociales, las humanidades y en algunos casos de las ciencias naturales y la ingeniería. Este es un punto fundamental a reconocer, dado que el profundizar sobre la propia reflexión o la observación de los procesos de construcción teórica de los estudios de la comunicación, presenta una problemática particular, la cual requiere un comentario más puntual que se desarrolla a continuación.

\section{ESTUDIAR A LA COMUNICACIÓN DESDE LA COMUNICACIÓN: LA NECESIDAD DE UN SEGUNDO ORDEN DE OBSERVACIÓN}

Para James Carey (1989), un problema básico de la comunicación es que al ser una experiencia cotidiana es en lo último en lo que reflexionamos por ser lo más aparente. Lo complicado entonces es pasar del mundo real y las cosas al lenguaje y su conceptualización. En este marco, estudiar comunicación implica el estudio del proceso social actual donde formas lógicas y marcos explicativos son creados, aprehendidos y usados, lo que genera inevitablemente un pensamiento circular, dado que en el estudio de la comunicación desde la misma comunicación, lo observado, el observador y la observación, se contienen a sí mismos. Según Carey, el resultado ha sido la generación 
de modelos que producen diferentes relaciones sociales, dado que los modelos no son sólo representaciones de la comunicación, sino representaciones "para" la comunicación, por lo que el estudio de la comunicación también comprende el indagar sobre la creación, aprehensión y usos de los modelos en sí mismos. Los modelos "crean" lo que nosotros ingenuamente creemos que "representan", por eso algunos se convierten en instituciones sociales. Sobre la comunicación se han construido entonces, modelos explicativos sobre su operación epistemológica (Lanigan, 1992; Martín Serrano, 2007; Piñuel \& Lozano, 2006), pero también teorías explicativas sobre su naturaleza ontológica (Lanigan, 1988) y principios axiológicos sobre su fundamentación práctica (Anderson, 1996; Craig, 1999, 2008a), al mismo tiempo que en el vivir comunicativo se construyen sistemas sociales y los propios principios explicativos. Emerge entonces la complejidad no como un obstáculo, sino como un argumento explicativo de la naturaleza misma del reflexionar sobre la comunicación a través de la comunicación (Aguado, 2003).

En este mismo sentido, en su emblemático trabajo sobre la teoría de la comunicación como campo, Robert T. Craig (1999) argumenta que la teoría de la comunicación es un área coherente de prácticas metadiscursivas, es decir, un campo discursivo sobre discursos que tienen implicaciones para la práctica de la comunicación. Por lo tanto, más que pensar en la idea de "una" teoría de la comunicación, Craig sugiere que es en la conjunción de una matriz general que acepte las diferentes visiones sobre la comunicación en donde reside la clave para el futuro de la teoría de la comunicación como campo de estudio. En este sentido, el equivalente del relativismo teórico reconocido en las primeras líneas de este artículo es lo que Craig denomina las "rutas de la incoherencia" en la teoría de la comunicación, desde donde reconoce que la vía para remediar la incoherencia no es la propuesta quimérica de "una teoría unificada de la comunicación", dado que en realidad ningún campo tiene una teoría semejante, lo cual no quiere decir que se suspenda toda búsqueda de bases comunes dentro de una matriz disciplinar compartida.

Es desde esta perspectiva que Craig (1999) sugiere que la clave es entender a la comunicación como el modo fundamental de explicación 
y no como un elemento subordinado a otro tipo de lógicas, lo cual implica no sólo desarrollar ese punto de vista particular, sino tomar en consideración el movimiento y el cambio natural del mundo social. Por lo tanto, no se trata de escoger entre un principio de transmisión (matemático o cibernético) o uno de corte interpretativo (semiótico, hermenéutico o fenomenológico) para fundamentar una matriz general para la teoría de la comunicación, sino, por el contrario, de elaborar una matriz que permita la inclusión de más de una visión sobre la comunicación, es decir, un lugar donde los distintos modelos teóricos de la comunicación puedan interactuar: un metamodelo o un modelo de segundo orden (Craig, 1999). El metamodelo que sugiere Robert T. Craig, pero que no desarrolla, parte de la crítica al supuesto de validez bajo el cual las teorías se construyen a partir del cuestionamiento de toda posición metadiscursiva, sin embargo, algunas teorías ponen en duda lo que en otras está dado por sentado, y cuyo proceso produce un juego autorreferencial del cual únicamente se puede salir cambiando el cuestionamiento de "cómo la comunicación es posible" a "cómo la comunicación es consumada en la práctica" y, desde el punto que aquí se sostiene, de "como la comunicación podría ser" si se le piensa desde marcos distintos, sobre todo si se toma en serio la afirmación de Craig en la que sugiere que la práctica técnica de la teoría de la comunicación generalmente deriva de nuestras prácticas ordinarias en las que hablamos sobre comunicación.

En síntesis, para Craig (1999), de lo que se trata es de reconstruir la teoría de la comunicación como un metadiscurso teórico comprometido en un diálogo con el metadiscurso práctico de la vida diaria, de esta forma, el metadiscurso teórico hace referencia a la discusión sobre el mérito de teorías alternativas. La comunicación tiene, entonces, el potencial de ser una disciplina práctica, lo cual, de ser cierto, se convierte en una herencia que la teoría de la comunicación esboza desde sus fundamentos. En esto radica entonces la necesidad de volver en el tiempo a los viejos problemas, recuperar el debate sobre la producción teórica en los estudios de la comunicación para reintegrar en él la práctica profesional de los sujetos y las estructuras epistemológicas de la formalización de conocimiento, para desde ahí construir alternativas y supuestos sobre lo que implica un cambio de concepción sobre 
el estudio de la comunicación, pero sobre todo, acerca de los procesos de producción teórica dentro del propio campo. ${ }^{5}$

\section{DE LA IDEA DE COMUNICACIÓN}

A LAS TEORÍAS DE LA COMUNICACIÓN:

\section{LA PARADOJA DE LOS LUGARES COMUNES}

En las secciones anteriores se ha puesto de manifiesto la necesidad de estudiar los procesos de producción teórica en los estudios de la comunicación con la finalidad de replantear tanto el problema del relativismo teórico como aquellos generados por la debilidad de la fundamentación teórica y epistemológica de las propuestas formales sobre la comunicación. De igual forma se han mostrado una serie de posturas que afirman la imposibilidad de regresar en el tiempo para plantear desde la reconstrucción histórica, una matriz organizativa general. Sin embargo, paralelamente se ha ido construyendo una segunda ruta desde donde se puede argumentar exactamente lo contrario, que es posible reconocer una ruta genealógica compartida que permite proponer bases comunes

5 En este mismo sentido, en el trabajo ya citado de Berger et al. (2010), los autores reconocen en primera instancia lo inoperable que resulta la separación de la producción teórica o la teoría en sí misma de la práctica de investigación donde los principios teóricos se operacionalizan en elementos empíricos específicos, y reconocen al mismo tiempo que la ciencia de la comunicación implica algo más que el simple uso de poderosos métodos de investigación, sofisticadas técnicas estadísticas o bien, algo más que la simple resolución de problemas comunicativos prácticos, dado que para los autores, la ciencia de la comunicación en realidad se preocupa por la identificación de regularidades sobre la propia comunicación y sus efectos y, más precisamente, se interesa en la explicación de por qué semejantes regularidades emergen en el mundo. Sin embargo, la tarea de la identificación de la emergencia de una regularidad se ve obstaculizada por la confusión en el uso que se le da a la teoría dentro de la literatura contemporánea de la investigación de la comunicación. Lo que emergen entonces no son regularidades comunicativas identificables, sino regularidades en la separación de la teoría de la sustancia que explica o bien, de la ilusión posible en una teoría sin sustancia. 
para la unificación de una matriz científica general, de una ciencia de la comunicación, de una comunicología, es decir, es posible desarrollar el proyecto que Robert T. Craig (1999) sugería una década atrás. Sobre esta posibilidad, un breve apunte.

En un exhaustivo trabajo reconstructivo sobre la idea de comunicación y la emergencia de lo que formalmente puede ser nombrado como "teoría de la comunicación", John Durham Peters (1999) plantea un mapa de relaciones históricas desde donde más tarde podrán ser reconocidas fuentes históricas y científicas del estudio de la comunicación (Galindo, 2008). En su trabajo, Peters (1999) sostiene que no es sino hasta finales de los años cuarenta, con la aparición de la Teoría Matemática de la Comunicación de Claude Shannon publicada en 1948, que el espacio conceptual de la comunicación se reorganiza. La teoría hablaba de algo que era familiar a lo que sucedía en la guerra, a las acciones de gobierno e inclusive a los fenómenos que sucedían en la vida diaria, y ese algo se sintetizó bajo el concepto de información, el cual dejó de ser un concepto que hacía referencia a una unidad de medida para convertirse en el principio de inteligibilidad del universo.

El punto fundamental de la transformación que generó en el mundo académico la aparición de la información como concepto constructor, implicó repensar las tesis que hasta el momento se habían mostrado, todo, en términos del intercambio de información. En palabras de Peters (1999), la "comunicación fue un concepto capaz de unificar las ciencias naturales (el DNA como el gran código), las artes liberales (el lenguaje como comunicación) y las ciencias sociales (la comunicación como el proceso social básico)" (p. 26). Este es un punto clave que no sólo Peters reconoce, sino que varios autores lo ponen al centro de la emergencia misma de la comunicación, de la historia de la comunicación y de la posible emergencia de un principio constructivo unificador (Escarpit, 1977; Mattelart \& Mattelart, 1997). La teoría matemática de la comunicación es entonces un primer gran referente sobre lo que la comunicación es y lo que describe, sin embargo, será la cibernética, la gran matriz epistemológica.

Para autores como Manuel Martín Serrano (1990), la emergencia no de una teoría de la comunicación sino de una epistemología de la comunicación, tiene como contexto sociohistórico los años cuarentas en Estados Unidos y como fundamento epistemológico la Teoría Matemática 
de la Comunicación de Claude Shannon (1948/1979) y la Cibernética propuesta por Norbert Wiener (1960). Lo anterior no quiere decir que la comunicación como concepto, como palabra o como objeto de estudio no existiese en otras ciencias y disciplinas, sino que faltaba un hilo conductor que lograra integrar esas muchas propuestas, es decir, existía la necesidad de un saber integrado de los fenómenos físicos, cognitivos, biológicos, tecnológicos, sociológicos y psicológicos. En palabras de Manuel Martín Serrano (1990):

El nuevo saber no se concebía como una suma de conocimientos, ni siquiera como la integración de saberes precedentes de las ciencias naturales, sociales y humanísticas. Consistió en la aplicación de otro punto de vista, cuya especificidad era la siguiente: organismos y organizaciones tan diversas tenían en común que se transformaban y transformaban su entorno, sin perder la organización que les diferenciaba de otros. Aquello que en cada uno de ellos aseguraba la permanencia, en el cambio, era precisamente la información. Los desarrollos de este paradigma serían las ciencias de la comunicación (p. 66).

Sin embargo, si bien la teoría matemática de la información y la cibernética tienen cierto reconocimiento en la historia de la teoría y epistemología de la comunicación, en realidad no son las más frecuentes ni en los procesos de construcción teórica ni en los estudios empíricos (Anderson, 1996; Bryant \& Miron, 2004; Galindo, 2008). Pero, ¿por qué si se reconocen ambos como fundamentos epistemológicos directos no funcionan como tales en los estudios de la comunicación? La respuesta a esta pregunta se encuentra en la historia misma que recupera Peters (1999), dado que si bien ambas perspectivas ponen en el mapa científico la palabra comunicación, son otras las que ponen tanto el método como las aproximaciones teóricas. Así, por ejemplo, en el trabajo ya citado de Robert T. Craig (1999), el autor reconoce siete tradiciones como puntos de partida para organizar la dimensión metadiscursiva del campo de la teoría de la comunicación, a saber:

1. La tradición retórica (la comunicación como el arte práctico del discurso).

2. La tradición semiótica (la comunicación como la mediación intersubjetiva de los signos). 
3. La tradición fenomenológica (la comunicación como la experimentación del otro).

4. La tradición cibernética (la comunicación como el procesamiento de información).

5. La tradición sociopsicológica (la comunicación como expresión, interacción e influencia).

6. La tradición sociocultural (la comunicación como la (re)producción del orden social).

7. La tradición crítica (la comunicación como reflexión discursiva).

En un intento similar, Miquel Rodrigo Alsina (2001) reconoce al menos tres grandes perspectivas en la historia de la teoría de la comunicación, a saber:

1. La perspectiva interpretativa (Escuela de Palo Alto, interaccionismo simbólico y construccionismo).

2. La perspectiva funcionalista.

3. La perspectiva crítica (Escuela de Frankfurt, la economía política y los estudios culturales).

Por su parte, para construir un fundamento teórico de lo que denomina las "teorías de la comunicación digital interactiva" y en un intento por diferenciar las teorías de la comunicación de masas de aquellas cuyo énfasis está puesto en la comprensión de las hipermediaciones generadas en los espacios virtuales, Carlos Scolari (2008) propone igualmente cinco paradigmas fundacionales de las teorías de la comunicación:

1. El paradigma informacional (ofrecía a los sociólogos un modelo sencillo para representar lo que para ellos era un proceso lineal y directo que iba de un emisor a un receptor y la subsecuente tuba de Schramm).

2. El paradigma crítico (apoyado en la Escuela de Frankfurt, la economía política de corte marxista y el psicoanálisis).

3. El paradigma empírico-analítico (en oposición al paradigma crítico y fundamentado en el modelo de la Mass Communication Research que se puede ver como una confrontación entre un modo europeo y otro estadounidense que habla sobre la comunicación de masas). 
4. El paradigma interpretativo-cultural (entiende a la comunicación de masas como una construcción social).

5. El paradigma semiótico-discursivo (centrado en los trabajos de Saussure y Peirce y sus subsiguientes desarrollos en Roland Barthes, Umberto Eco y Paolo Fabbri, entre muchos otros).

En un sentido distinto, en México, el Grupo Hacia una Comunicología Posible (GUCOM) realizó igualmente su propuesta de las fuentes históricas y científicas que se encuentran en la base la construcción conceptual en los estudios de la comunicación, reconociendo nueve fuentes:

1. La sociología funcionalista.

2. La sociología fenomenológica.

3. La sociología crítica.

4. La sociología cultural.

5. La economía política.

6. La psicología social.

7. La semiótica.

8. La lingüística.

9. La cibernética (Galindo, 2008). ${ }^{6}$

Por otro lado, Oliver Boyd-Barrett (2006) en su propuesta de un modelo para la investigación y la enseñanza de los medios en el marco de un mundo globalizado, recupera lo que considera son los logros más importantes de la investigación de la comunicación anglo-americana de los últimos 50 años, los cuales agrupa dentro de tres grandes categoría:

1. Los estudios culturales.

2. La economía política.

3. Los estudios de la globalización.

6 Recientemente, Carlos Fernández y Laura Galguera (2009) han propuesto una organización muy similar a la sugerida por el GUCOM reconociendo seis "tradiciones" en el estudio de la comunicación: la tradición semiótica, fenomenológica, cibernética, sociopsicológica, sociocultural, crítica y retórica. Dentro de cada una de estas tradiciones los autores ubican diferentes autores y teorías particulares. 
Como es posible observar, más allá de los nombres y las agrupaciones que cada propuesta realiza, es posible reconocer algunas rutas compartidas y lugares comunes, pero junto con el reconocimiento de las similitudes es posible asumir que si bien ninguna de ellas en realidad ha funcionado como matriz general, existen pistas de lo que se puede hacer con ellas en el futuro. Sin embargo, la paradoja es que, mientras es posible reconocer algunos lugares comunes en la historia de la comunicación, de las teorías de la comunicación y de la epistemología de la comunicación, como se ha mostrado, ninguno de los paradigmas, tradiciones, perspectivas o fuentes históricas y científicas es admitida en la práctica como una ciencia integral de la comunicación o como una matriz teórica general, como tampoco se asume como propio de la comunicación el objeto de conocimiento que cada una de ellas pone al centro. Por lo tanto, más que cerrar el debate en la imposibilidad de semejante marco, vale la pena explorar algunas de las propuestas que han caminado hacia la unificación teórica y hacia el esclarecimiento del objeto mismo de la reflexión de la comunicación. Dos de estas propuestas se analizan brevemente a continuación.

\section{DE LA COMUNICACIÓN COMO PRINCIPIO EXPLICATIVO}

\section{A LA COMUNICACIÓN COMO DISCIPLINA PRÁCTICA}

Hasta este punto se han mostrado algunas consecuencias prácticas del uso de las teorías de la comunicación y de la emergencia del relativismo teórico, de igual forma se han mostrado algunos argumentos sobre la imposibilidad de una teoría unificadora de la comunicación, los cuales han sido contrastados con aquellos postulados explícitos sobre las posibilidades de reconocer fuentes históricas y científicas compartidas desde donde es posible proponer una matriz epistemológica general. Por lo tanto, las siguientes líneas muestran dos propuestas concretas desde donde es posible continuar la discusión, una que reconoce que el futuro de la comunicación depende de que sea comprendida como una disciplina práctica y otra que propone de entrada una matriz teórica general, una ciencia integral de la comunicación. Se partirá, por tanto, del planteamiento de la primera posibilidad.

En un texto reciente, Robert T. Craig (2008a) reconoce que: 
Lo que principalmente explica la emergencia disciplinaria del campo es la significante relación de la comunicación como una categoría de práctica social y es, a través de la reconstrucción de sus tradiciones intelectuales alrededor de esa categoría, que el campo puede esperar volverse no sólo más productiva e intelectualmente coherente, sino más útil socialmente (p. 7).

La idea es, por tanto, que una teoría sobre la disciplinarización de un campo deriva su identidad y coherencia de su participación en la conversación de las disciplinas, así:

El carácter específico de la comunicación como disciplina, puede ser entendido en términos de su contribución al conocimiento en determinadas tradiciones intelectuales, en la evolución de sus formas institucionales y en su relevancia hacia la "comunicación" entendida como una categoría socioculturalmente constituida de problemas y prácticas (p. 7).

Para Craig, en realidad las disciplinas son una comunidad conversacional con una tradición de argumentación particular, la cual participa de una comunidad conversacional más amplia con sus propias tradiciones de argumentación, por lo que dichas disciplinas no se encuentran fundadas sobre categorías fijas de conocimiento, sino que son formaciones discursivas que emergen, evolucionan, se transforman y disipan en la continua conversación entre disciplinas, por lo que su fuerza radica en la resonancia de su discurso a través de la sociedad, en su distinción intelectual y su productividad así como en su consolidación en los esquemas institucionales existentes de organización (institucional, intelectual y cultural). Lo anterior puede convertir a una disciplina en una "disciplina práctica" cuya importancia está dada por la creencia en que sus principios pueden ayudar a resolver problemas prácticos ya reconocidos socialmente. Sin embargo, esto no es lo que ha sucedido con el campo de estudio de la comunicación, pues para Craig (2008a):

Si la comunicación es ahora una disciplina, es porque los académicos de la comunicación han aprovechado una oportunidad retórica. Influenciando el sentido común con la relevancia de su tema han ganado acceso a los recursos 
institucionales e intelectuales que han adaptado y transformado en términos de "problemas de comunicación" en la sociedad (p. 10).

Nuevamente, el problema se sintetiza en la relación entre fundamentación conceptual e institucionalización, problema en donde el relativismo teórico emerge como un síntoma claro de lo complicado que ha resultado formular puentes entre ambas.

Como se puede apreciar, desde la propuesta de Craig (2008a), las vías para la subsistencia de la comunicación como disciplina, más allá del reconocimiento de los problemas epistemológicos que genera el uso indiferenciado de principios teóricos, es su vitalidad como disciplina práctica. Sin embargo, es importante puntualizar que la propuesta no supone que haya disciplinas no prácticas o que conocer el mundo sea poco práctico, sino que la comunicación debe comprenderse en sí misma como una práctica específica. De ahí que Craig (2006) proponga que, para que la comunicación sea considerada una práctica, debe de haber un concepto cultural de la comunicación que haga referencia al tipo general de prácticas en las que la gente se involucra sin importar lo que comunican, por lo tanto, afirmar que la comunicación es una práctica en nuestra cultura, significa no sólo asumir que nos comunicamos o que tenemos prácticas comunicativas, sino que la práctica de la comunicación se ha convertido en una práctica significativa. De ahí que no haya una carga positiva o negativa sobre la práctica misma de la comunicación, sino una autoconciencia y reflexión sobre la comunicación de la que derivan metadiscursos (discursos sobre discursos) referentes a prácticas metadiscursivas o formas de referirse a la comunicación con propósitos prácticos. Según Craig (2006), es este metadiscurso el que se ha expandido y especializado en nuestras sociedades al grado de convertirse en un discurso normativo formando así una disciplina académica que desarrolla sus propias prácticas metadiscursivas altamente sofisticadas, que es otra forma de nombrar al campo de las teorías de la comunicación y una forma de cultivar la práctica de la comunicación en nuestra sociedad.

Sin embargo, en contraposición al argumento de Craig, lo que aquí se argumenta es que los problemas de la dimensión conceptual de la comunicación no tienen únicamente relación con su dimensión práctica, 
sino con problemas en la base misma de los procesos de producción de conocimiento en el propio campo, es decir, con la práctica de investigación, un tipo particular de práctica comunicativa. Así que una segunda opción no implica caminar en el sentido de una disciplina práctica, sino en el sentido de una teoría unificada, y un ejemplo de esta ruta es el trabajo que ha venido desarrollando las últimas dos década el danés Soren Brier (2008), quien de hecho comparte algunos de los problemas aquí planteados. Para Brier, la debilidad de los estudios comunicativos e informacionales tradicionales basados en teorías sobre los flujos de información o los datos en sí, han hecho emerger problemas en lo que respecta a la forma en que los sistemas de conocimiento son construidos y organizados, es decir, han hecho emerger problemas en el nivel epistemológico propiamente que no se resuelven cambiando nuevamente la discusión como lo hiciera Schramm 50 años atrás, sino haciéndoles frente en el mismo nivel.

De esta forma, el mismo Brier (2008), basado en la propuesta de la cibernética de segundo orden y la semiótica, plantea que es posible desarrollar nuevos conceptos que ayuden a entender y desarrollar sistemas sociales como redes auto-organizadas y auto-reproducidas, por lo tanto, en vez de hablar en términos de "comunicación de información" la propuesta es hablar en términos de "significados conjuntamente actualizados". Para Brier, las ciencias de la información, en lo que respecta a los sistemas vivos y a los sistemas humanos, no son capaces de explicar aspectos vitales del fenómeno de la comunicación y la cognición como lo es la emergencia del significado en los ámbitos limitados de los contextos sociales y en los ámbitos generales de la reproducción y supervivencia de los seres vivos. Aparece entonces el problema del significado en el marco del punto de vista mecanicista que brinda la teoría de la información y la cibernética en el marco general de las ciencias de la información, dado que dicha visión se extiende a la comprensión del conocimiento, la naturaleza, el lenguaje y, finalmente, a la conciencia humana. En consecuencia, para Brier (2008), el paradigma del procesamiento de información nunca tendrá éxito en describir los problemas fundamentales en la mediación semántica del contenido de un mensaje de un productor a un usuario, dado que es incapaz de tomar en consideración los aspectos 
fenomenológicos y sociales de la cognición. Por lo tanto, la idea de unir a la semiótica peirceana con la cibernética de segundo orden no sólo responde a un problema epistemológico, sino a una oportunidad de expandir los horizontes de observación, tanto de lo que se observa como del sistema que lo hace y al mismo tiempo, de convertirse en una propuesta teórica que pone al centro a la comunicación y su propio proceso de construcción teórica. En palabras de Brier (2008):

Los dos marcos transdisciplinarios de la cibernética de segundo orden y la semiótica tríadica de Peirce aparecen como promisorias para desarrollar un diálogo entre los sistemas sociales [Luhmann] y el conocimiento sobre la cognición y la producción de significación en los sistemas biológicos [autopoiesis y acoplamiento estructural]. La cibernética de segundo orden ha abandonado la idea objetivista de la información pero no ha desarrollado todavía un concepto de signo. La semiótica estudia científicamente a la significación como dimensión básica y universal de la realidad humana. La semiótica de Peirce también aborda los signos no intencionales y contiene un concepto evolutivo y orientado al proceso del signo triádico de segundo orden - en el cual todas las partes de la semiosis son signos. Sin embargo, carece de conocimiento sobre la auto-organización de la cognición y del acoplamiento estructural de los observadores. Así, se sugiere que estos dos marcos pueden ser integrados en algo como el concepto de los juegos del lenguaje de Wittgenstein y ese pre-lenguaje de los sistemas biológicos que producen significación pueden ser entendidos como juegos sígnicos. El significado comunicativo es generado por sistemas autopoiéticos en juegos del lenguaje y sígnicos (p. 101).

De esta manera, Brier supone que una teoría consistente de la información, la cognición y la comunicación debe necesariamente comprender las ciencias sociales y las humanidades, así como las ciencias biológicas y de lo psicoquímico. El problema del sentido y la significación en los sistemas vivos hizo evidente algunos límites en la cibernética, pero también apuntó la necesidad de relacionarse con otras ciencias, con otras epistemologías. De esta forma emerge la necesidad de relacionarse con la semiótica, de la cual deviene un marco de integración, el marco de la cibersemiótica, el cual podría ser sintetizado 
como la búsqueda de las rutas biológicas, psíquicas y sociales de la necesidad humana y biológica del significado y la auto-organización en sus procesos de conocer/observar el mundo y en la formulación de las explicaciones que sobre él se hagan. La cibersemiótica se presenta a sí misma como una nueva visión no reduccionista de la cognición y la comunicación a partir de los desarrollos de la biosemiótica, lo que supone implícitamente la extensión del entendimiento de la evolución de la información en animales, máquinas y humanos, así como en los procesos de organización y auto-organización sistémica. Al plantear como objetivo central una ciencia integral de la información, la cibersemiótica se plantea como una visión interdisciplinar que integra distintos marcos en un nivel meta-discursivo que le da nacimiento a una visión diferente no sólo de la vida y los procesos cognitivos, sino de la comunicación y su construcción epistemológica. Puede ser, por tanto, la matriz general que planteaba Robert T. Craig una década atrás.

Propuestas como las mostradas hacen posible argumentar que una ciencia general de la comunicación, una matriz general de organización teórica o una comunicología son proyectos posibles, dado que, como se ha mostrado, existen propuestas que han caminado en ese sentido. Sin embargo, lo que no es posible decir es que su desarrollo y emergencia resolverá el problema del relativismo teórico en los estudios de la comunicación, dado que este problema depende de la práctica de investigación, del uso de la teoría y no tanto de su solidez epistemológica. Lo anterior permite observar que las paradojas generadas por los problemas no resueltos han alcanzado su límite operativo y han comenzado a generar efectos negativos para el campo de estudio de la comunicación como son las "rutas de la incoherencia" la "erosión epistemológica" el "pluralismo teórico indiferenciado" o el relativismo teórico, todos términos que nombran una misma problemática. Pero conjuntamente con la gran cantidad de propuestas que han reconocido el mismo problema y que han hecho propuestas para hacerle frente, es posible plantear escenarios alternativos, así que quizás es tiempo de recuperar todas esas propuestas y ponerlas a dialogar como lo han propuesto Berger et al. (2010), no para decir cómo es y cómo opera la producción teórica y la investigación empírica de la comunicación o cómo debería operar, sino para plantear rutas alternas, es decir, para explorar cómo podría ser su 
operar en el futuro, para plantear escenarios posibles. Sin embargo, para construir ese futuro todavía hay que clarificar por lo menos los niveles del debate, tema que se desarrolla a continuación.

\section{DE LAS TEORÍAS DE LA COMUNICACIÓN}

COMO CAMPO A LA METAREFLEXIÓN SOBRE

LA COMUNICACIÓN, ¿POR DÓNDE CONTINUAR EL DEBATE?

Según lo anotado en las secciones precedentes, dos de las discusiones más importantes en torno a las teorías de la comunicación y a la consideración científica del campo la comunicación tienen que ver con la posibilidad de plantear a la comunicación como un principio explicativo -lo que supondría una matriz teórica general- y la consideración de la comunicación como disciplina práctica, sin embargo, el problema no es tan simple, dado que entre una y otra hay elementos intermedios que requieren un comentario más puntual, dado que es de la forma en que se ha dado la relación entre la formulación conceptual de la comunicación y la práctica de investigación en donde es posible identificar uno de los retos más importantes que presenta el relativismo teórico en los estudios de la comunicación.

En un intento por organizar el proceso de construcción epistemológica en los estudios de la comunicación en términos de "niveles discursivos de semiosis científica", Klaus Bruhn Jensen (1995)7 planteaba un criterio de diferenciación entre el discurso de todos los días, el analítico, el metodológico, el teórico y el epistemológico, mostrando la importancia de reconocer los niveles de abstracción en la producción teórica. Lo importante de este primer antecedente es que, más allá del discurso propiamente, lo que es pertinente reconocer son los niveles de abstracción en los propios procesos de producción de conocimiento en toda empresa científica. Es evidente que, pese a que el conocimiento o discurso de todos los días es conocimiento o discurso finalmente, su nivel de abstracción y el objeto de conocimiento al que hace referencia es completamente diferente. Pensar entonces en

7 Para una visualización esquemática de la propuesta de Klaus Bruhn Jensen véase su trabajo, The Social Semiotics of Mass Communication (1995). 
una ciencia integrativa de la comunicación (Brier, 2008), en las teorías de la comunicación como campo (Craig, 1999), en una comunicología (Galindo, 2008; Lanigan, 1992) o en la comunicación como una disciplina práctica (Craig, 2008a) requiere necesariamente de la clarificación del nivel de abstracción de cada propuesta $\mathrm{y}$, sobre todo, del objeto de referencia de toda construcción conceptual que de ella pueda derivar. Así, emulando la propuesta que planteaba Jensen una década atrás, aquí lo que se propone es una ruta de reflexión que parte del reconocimiento de tres niveles de abstracción desde donde se puede organizar el mapa conceptual de la comunicación y de la cual es posible derivar una primera hipótesis sobre la emergencia del relativismo teórico en los estudios de la comunicación.

En principio, es fundamental hacer notar que toda abstracción se encuentra necesariamente vinculada a un problema, un objeto de observación o un objeto de conocimiento del cual derivan: a) un primer nivel de abstracción, b) un nivel teórico y c) un nivel epistemológico. En lo que respecta al primer nivel de abstracción, éste supone nombrar el fenómeno o el problema que está siendo observado pero no implica su formalización dentro de un sistema conceptual, lo cual requiere necesariamente del paso de la primera abstracción al nivel teórico. En este segundo nivel, el objeto de la reflexión ya se ha movido, dado que no implica la reflexión sobre el fenómenos en sí, sino sobre la naturaleza de lo que el primer nivel de abstracción ha puesto en forma al nombrar un fenómeno determinado. Como lo menciona Jesús Galindo (2009):

Mucho de lo que se desea nombrar ya está localizado en un campo semántico que le da sentido; se trata de averiguar sobre esos campos semánticos y definir los huecos de información existentes, las relaciones no establecidas, las partes no nombradas, los antecedentes y los consecuentes no explicitados, los marcos de contextualización no presentes (p. 152).

Es el paso de los fenómenos a los objetos de investigación y los objetos propios de la ciencia, es decir, se trata de pasar del sentido común a los sistemas conceptuales o los campos semánticos ya reconocidos. Sin embargo, si bien este segundo nivel tiene una cualidad conceptual, depende por completo de la dimensión empírica que lo ha motivado. 
Según Galindo (2009), "lo que llamamos teoría es un espacio conceptual de representaciones de referente empírico, al cual también se puede volver en una actitud de intervención en una perspectiva menos científica y más de ingeniería" (p. 152). Posteriormente lo que emerge es un tercer nivel de observación, ya no sobre el fenómeno o sobre su primera abstracción, sino sobre la teoría que pretende dar cuenta de él, es decir, el objeto de conocimiento es la teoría en sí, la cual ya tiene una distancia considerable con el fenómeno o problema que lo ha detonado en un primer momento. Se trata entonces del tercer nivel, el nivel propiamente epistemológico.

De esta manera, cada nivel se asocia con una posibilidad organizativa. El nivel epistemológico tiene que ver con las propuestas integrativas, con las propuestas de la comunicación como una matriz teórica general o con la posibilidad de una ciencia de la comunicación, de una comunicología (Galindo, 2008; Lanigan, 1992). Por su parte, el segundo nivel asociado a la teoría y, en este caso, a las teorías de la comunicación, se vincula con la posibilidad de construir una comunidad argumentativa a su alrededor, pero no tiene que ver con la dimensión ontológica de la comunicación ni con una ciencia integrativa, por lo tanto, es el lugar que ocupa la teoría de la comunicación como campo (Craig, 1999). Por otro lado, el primer nivel de abstracción puede ser considerado como una operación primaria en la práctica misma de investigación, es el lugar que Robert T. Craig (2006a, 2008a) le da a la comunicación como disciplina práctica. Por fines expositivos, lo anterior se sintetiza en la figura 1.

Además de permitir identificar el lugar que a cada nivel de abstracción le corresponde, la propuesta esquemática permite plantear algunas alternativas. Como se puede observar, el diálogo entre cada uno de los niveles es sumamente estrecho. Por ejemplo, el grado de proximidad entre el nivel epistemológico y teórico permite una relación dialógica más estrecha, sin embargo, es posible imaginar la dificultad que representa establecer un diálogo entre el nivel epistemológico y el primer nivel de abstracción de todo objeto de conocimiento o fenómeno de estudio. Por lo tanto, la hipótesis que aquí se sostiene con base en lo anteriormente mostrado, es que el relativismo teórico es un efecto que ha emergido en los estudios de la comunicación producto de la confusión 


\section{FIGURA 1}

NIVELES DE ABSTRACCIÓN Y REFLEXIÓN TEÓRICA EN LOS ESTUDIOS DE LA COMUNICACIÓN

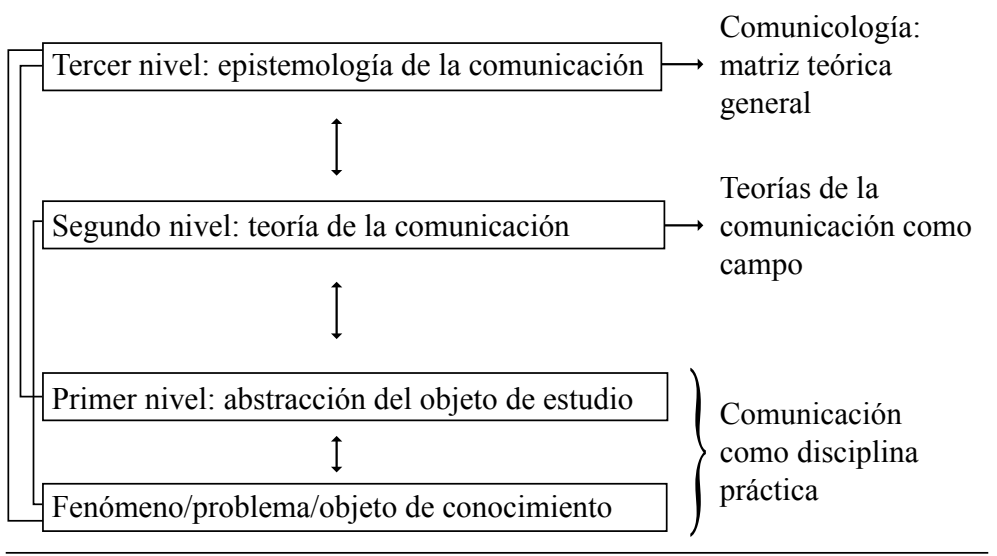

que se genera al considerar al primer nivel de abstracción como una dimensión teórica a priori. El resultado es la imposibilidad de reconocer sistemas conceptuales, principios teóricos o marcos epistemológicos en los trabajos empíricos de investigación. Resulta entonces comprensible por qué las propuestas de una matriz teórica general (Brier, 2008; Martín Serrano, 2007) o una comunicología (Galindo, 2008; Lanigan, 1992) resultan irrelevantes para la práctica de investigación en los estudios de la comunicación e, incluso, la propia reflexión de la teoría de la comunicación como un campo autónomo (Craig, 1999) pierde toda importancia académica.

De lo que se trata entonces es de la formulación de lazos que permitan integrar esos niveles ahora separados, es decir, de trazar los puentes que permitan la construcción de los primeros niveles de abstracción a partir de principios teóricos y epistemológicos fundamentales para la construcción de conocimiento en la práctica de investigación en los investigadores de la comunicación, lo que podría incluso volver pertinentes las propuestas teóricas y epistemológicas que se han formulado sobre la comunicación y que permanecen hoy en día separadas de la práctica misma. De esta manera, es posible entonces hablar de "subcampos" de la comunicación. 
Por ejemplo, para David Seibold (2008), la investigación aplicada de la comunicación -lo que correspondería a la aplicación del nivel teórico descrito en el esquema anterior- es una forma de comunicación académica que enfatiza la creación de conocimiento sobre comunicación en contextos específicos aplicable a distintos temas sociales y frecuentemente para la solución de problemas sociales. Si bien la investigación es guiada teóricamente o tiene la posibilidad de reformular principios teóricos, la construcción de teoría no es ni el propósito inmediato ni el principal en la investigación aplicada en los estudios de la comunicación, sino propiamente la relevancia social de su aplicación. La aplicación de la investigación de la comunicación es considerada entonces como un subcampo de la disciplina que reconoce a la teoría y a la práctica como elementos autorecursivos pero rechaza su equiparación.

Este grado de claridad sobre los niveles de reflexión puede ser una gran herramienta que potencie tanto la investigación en comunicación como el debate teórico y metodológico sobre el propio quehacer científico, los marcos usados para la reflexión, sobre esta misma, el papel del sujeto en los procesos de observación y, finalmente, la viabilidad de pensar en una ciencia integrativa de la comunicación, en un campo de las teorías de la comunicación, en una comunicología o en la comunicación como una disciplina práctica. Se trata entonces de tejer los puentes entre los niveles abstractos de construcción conceptual, pues a seis décadas de la discusión entre Bernard Berelson (1959) y Wilbur Schramm, David Riesman y Raymond Baver (1959), la historia es muy diferente, dado que el espacio académico contemporáneo se ha fragmentado a tal grado que podríamos hablar que en él habitan de manera separadas cuatro subcampos identificados con cada nivel mostrado en el esquema anterior, lo cuales no dialogan entre sí. El resultado es un relativismo teórico que dificulta enormemente caminar en cualquiera de las direcciones aquí sólo mencionadas e, incluso, dificulta el diálogo académico dentro del propio campo. La forma en que se investiga sobre la comunicación y la forma en que se construye conocimiento sobre la comunicación, paradójicamente, dificulta la comunicación en el campo de estudio de la comunicación.

Finalmente, los retos futuros para cualquiera de las posturas aquí mostradas no sólo es su consistencia teórica intrínseca, sino la clarifi- 
cación del lugar que ocupan en un diálogo mucho mayor que no sólo tiene que ver con la reflexión sobre la teoría de la comunicación, la consideración de la comunicación como una disciplina científica, la comunicación como principio explicativo o sobre la comunicología, sino con la práctica cotidiana de la investigación de la comunicación $\mathrm{y}$, especialmente, con la práctica misma del comunicar cotidiano. Como se puede observar, el reto es enorme, pero también es mucho el camino ya recorrido, por lo tanto, ya sea que se le llame comunicología, matriz constructiva general o campo de las teorías de la comunicación a este movimiento, la tarea constructiva, reconstructiva, crítica y reflexiva sobre la teoría de la comunicación y el problema del relativismo teórico, aparecen para las nuevas generaciones como una oportunidad y como un horizonte necesario e impostergable.

\section{Bibliografía}

Aguado, J. M. (2003). Comunicación y cognición. Bases epistemológicas de la complejidad. Sevilla: Comunicación Social, Ediciones y Publicaciones.

Anderson, J. A. (1996). Communication theory. Epistemological foundations. Nueva York: The Guilford Press.

Berelson, B. (1959). The state of communication research. Public Opinion Quarterly, 23, 1-6.

Berger, C., Rolof, M. \& Roskos-Ewoldsen, D. (2010). What is communication science? En C. Berger, M. Rolof \& D. Roskos-Ewoldsen (Ed.), Handbook of communication science (2. ed., pp. 3-20). Los Angeles/Londres/Nueva Delhi/Singapore/Washington: Sage Publications.

Boyd-Barrett, O. (2006). Publishing research and communications curriculum under globalization. En K. Lueng \& Lee (Eds.), Global trends in communication education and research (pp. 235-259). Cresskill, NJ: Hampton Press.

Brier, S. (2008). Cybersemiotics. Why information is not enough. Toronto/Buffalo/Londres: University of Toronto Press.

Bryant, J. \& Miron, D. (2004). Theory and research in mass communication. Journal of Communication, 4 (54), 662-704. 
Carey, J. W. (1989). Part I. Communication as culture. En J. W. Carey, Communication as culture. Essays on media and society (pp. 13110). Nueva York/Londres: Routledge.

Craig, R. T. (1999). Communication theory as a field. Communication theory, a Journal of the International Communication Association, 9, 119-161.

Craig, R. T. (2006). Communication as practice. En G. Shepherd, J. St. John \& T. Striphas (Eds.), Communication as ... perspectives on theory (pp. 38-47). Thousand Oaks, CA/Londres/Nueva Delhi: Sage Publications.

Craig, R. T. (2008a). Communication in the conversation of disciplines. Russian Journal of Communication, 1 (1), 7-23.

Craig, R. T. (2008b). Communication as a field and discipline. En W. Donsbach (Ed.), The international encyclopedia of communication (Pp. 675-688). Inglaterra: Blackwell Publishing.

Dance, F., \& Larson, C. (1976). The functions of communication: A theoretical approach. Nueva York: Holt, Rinehart and Winston.

Donsbach, W. (2006). The identity of communication research. Journal of Communication, 3 (56), 437-448.

Escarpit, R. (1977). Teoría general de la información y la comunicación. Barcelona: Icaria Editorial. [Trabajo original publicado en 1976].

Fernández, C. \& Galguera, L. (2009). Teorías de la comunicación. Madrid: McGraw-Hill Interamericana.

Galindo, J. (Coord.) (2008). Comunicación, ciencia e historia. Fuentes cientificas históricas hacia una comunicología posible. Madrid: McGraw Hill-Interamericana.

Galindo, J. (2009). Relación entre problemas y preguntas. Apuntes para un taller de investigación en comunicación y cultura. Estudios sobre las Culturas Contemporáneas, 29 (15) 149-160. Época 2.

Gerbner, G. (1983). The importance of being critical - In one's own fashion. Ferment in the Field, Journal of Communication, 3 (33), 355-362.

Herbst, S. (2008). Disciplines, intersections, and the future of communication research. Journal of Communication, 4 (58), 603-614. 
Jensen, K. B. (1995). The social semiotics of mass communication. Londres/Thousand Oaks, CA/New Delhi: Sage Publications.

Krippendorff, K. (1994). The past of communication's hoped-for future. En Levy \& Gurevitch (Eds.), Defining media studies. Reflections on the future of the field (pp. 45-52). Oxford/Nueva York: Oxford University Press.

Kuhn, T. S. (2006). La estructura de las revoluciones científicas. México: FCE. (Trabajo publicado en 1962).

Lanigan, R. (1988). Phenomenology of communication. MerleauPonty's thematics in communicology and semiology. Pittsburgh, Pennsylvania: Duquesne University Press.

Lanigan, R. (1992). The human science of communicology. Pittsburgh, Pennsylvania: Duquesne University Press.

Martín Serrano, M. (1990). La epistemología de la comunicación a los cuarenta años de su nacimiento. TELOS. Cuadernos de comunicación, tecnología y sociedad, 22, 65-75.

Martín Serrano, M. (2007). Teoría de la comunicación. La comunicación, la vida y la sociedad. Madrid: McGraw-Hill Interamericana.

Mattelart, A. \& Mattelart, M. (1997). Historia de las teorías de la comunicación. Barcelona: Paidós.

Maturana, H. \& Varela, F. (2006). El árbol del conocimiento. Santiago de Chile: Editorial Universitaria.

Peters, J. D. (1986). Institutional sources of intellectual poverty in communication research. Communication Research, 4 (13), 527-559.

Peters, J. D. (1988). The need for theoretical foundations. Replay to Gonzalez. Communication Research, 3 (15), 309-317.

Peters, J. D. (1999). Speaking into the air. A history of the idea of communication. Chicago/Londres: The University of Chicago Press.

Piñuel, J. L. \& Lozano, C. (2006). Ensayo general sobre la comunicación. Barcelona: Paidós.

Rodrigo Alsina, M. (2001). Teorías de la comunicación. Ámbitos, métodos y perspectivas. Barcelona: UAB/U-Jaume I/U.P. Fabra/U. Valencia.

Scolari, C. (2008). Hipermediaciones. Elementos para una teoría de la comunicación digital inter-activa. Barcelona: Gedisa. 
Searle, J. (1997). La construcción de la realidad social. Barcelona: Paidós. (Trabajo publicado originalmente en 1995)

Seibold, D. R. (2008). Applied communication research. En W. Donsbach (Ed.). The International Encyclopedia of Communication (Pp. 189-194) Inglaterra: Blackwell Publishing.

Schramm, W. (1963). The science of human communication. Nueva York: Basics Books.

Schramm, W. (1983). The unique perspective of communication: A retrospective view. Ferment in the Field, Journal of Communication, 3 (33), 6-17.

Schramm, W., Riesman, D. \& Bauer, R. (1959). The state of communication research: Comments. Public Opinion Quarterly, 23, 6-17.

Shannon, C. (1979) Teoría matemática de la comunicación. México: SCT. (Trabajo original publicado en 1948)

St. John, J., Striphas, T., \& Shepherd, G. (2006). Introduction: Taking a stand on theory. En J. St. John, T. Striphas \& G. Shepherd, Communication as... Perspectives on Theory (Pp.XI-XIX). Thousand Oaks, CA: Sage.

Vidales, C. (2008). La semiótica/semiología como fuente histórica y científica de una comunicología posible. En J. Galindo (Coord.), Comunicación, ciencia e historia. Fuentes cientificas históricas hacia una comunicología posible (pp. 343-408). Madrid: McGraw Hill-Interamericana.

Vidales, C. (2009a). La semiótica como matriz de estructuración de las teorías de la comunicación. En E. Tarasti, Communication: Understanding / Misunderstanding, Vol 3. Procedings of the 9th Congres of the IASS/AIS (Pp. 1884-1892). Finlandia: Acta Semiotica Fenica XXXIV/International Semiotic Institute/Semiotics Society of Finland.

Vidales, C. (2009b enero/junio). La relación entre la semiótica y los estudios de la comunicación: un diálogo por construir. Comunicación y Sociedad, 11, 37-71. Nueva época. México: UdeG.

Vidales, C. (2009c). La sociosemiótica y la comunicología histórica. La organización biológica y social de la semiosis. En J. Galindo (Coord.), Sociología y comunicología. Historias y posibilidades (pp. 219-266). Argentina: EUCASA. 
Vidales, C. (2010). Semiótica y teoría de la comunicación. Tomo 1. México: CAEIP.

Vidales, C. (2011). Semiótica y teoría de la comunicación. Tomo 2. México: CAEIP.

Wallerstein, I. (2005). Las incertidumbres del saber. Barcelona: Gedisa [Trabajo original publicado en 2004].

Wiener, N. (1960) Cibernetica. Madrid: Guadalajara de Publicaciones. 